\title{
Upregulation of circular RNA circ-FAM53B predicts adverse prognosis and accelerates the progression of ovarian cancer via the miR-646/VAMP2 and miR-647/MDM2 signaling pathways
}

\author{
DAN SUN $^{1}$, JIANING LIU ${ }^{1}$ and LILI ZHOU ${ }^{2}$ \\ Departments of ${ }^{1}$ Gynecology, and ${ }^{2}$ Gynecology and Obstetrics, \\ Heilongjiang Provincial Hospital, Harbin, Heilongjiang 150000, P.R. China
}

Received May 31, 2019; Accepted September 3, 2019

DOI: $10.3892 /$ or.2019.7366

\begin{abstract}
Ovarian cancer (OC) is a common cancer of the human genital system. Circular RNAs (circRNAs) play an important role in carcinogenesis and progression of various cancers. The present study aimed to clarify the expression profile and functions of circ-FAM53B in the progression of $\mathrm{OC}$ and reveal its underlying mechanisms. Relative levels of circ-FAM53B in OC specimens and cell lines were determined by quantitative real-time polymerase chain reaction (qRT-PCR). The clinical significance of circ-FAM53B in OC patients was analyzed through Fisher's exact test, Kaplan-Meier curves, and Cox regression analysis. Subsequently, the regulatory effects of circ-FAM53B on the proliferative, apoptotic, migratory, and invasive potential of OC cells were determined by loss/gain-of-function experiments. Mechanistically, bioinformatics analysis and luciferase reporter gene assay were used to reveal the potential molecular mechanisms of circ-FAM53B in OC. circ-FAM53B was overexpressed in OC specimens and cells and correlated with clinical severity and poor prognosis of OC patients. The overexpression of circ-FAM53B accelerated the proliferation, migration, and invasion of HO8910 cells; however, it decreased the number of apoptotic cells. Silencing of circ-FAM53B induced the opposite effect. Through dual-luciferase reporter gene assay and functional experiments, the potential functions of circ-FAM53B/miRNA-646/vesicle-associated membrane protein 2 (VAMP2) and circ-FAM53B/miRNA-647/mouse double minute 2 (MDM2) in mediating the progression of OC were identified. Collectively, the present results indicated that circ-FAM53B could be a competing endogenous RNA (ceRNA) to competitively sponge miR-646 and miR-647 to
\end{abstract}

Correspondence to: Professor Jianing Liu, Department of Gynecology, Heilongjiang Provincial Hospital, Harbin, Heilongjiang 150000, P.R. China

E-mail: ljnchina@aliyun.com

Key words: ovarian cancer, circular RNA, circ-FAM53B, VAMP2, MDM2 upregulate VAMP2 and MDM2 expression at the post-transcriptional level, thus mediating the cellular behaviors of OC cells.

\section{Introduction}

Ovarian cancer (OC), a cancer of the genital system, is a common malignancy worldwide (1), and its incidence has been increasing in recent years (2). Radical surgery along with radio-chemotherapy is employed for treating OC (3). For most OC patients, the outcome of radical surgery followed by either chemotherapy or radiotherapy is not favorable $(4,5)$. Thus, there is an urgent need to find more effective therapeutic targets for treating OC.

Circular RNAs (circRNAs) are a type of non-coding RNAs (ncRNAs), which do not have a potential to code for proteins $(6,7)$. Some of them are critically dysregulated in cells and are associated with disease progression in humans (8-12). Recently, disordered circRNAs were identified in many tumors, including OC (13-16). For instance, circP4HB promoted aggressiveness and metastasis of non-small-cell lung carcinoma (NSCLC) by absorbing miR-133a-5p (17). Regarding OC, circRNA profiling revealed circRNA1656 as a novel biomarker in high-grade serous OC (18). To date, various circRNAs have been identified, but only a few circRNAs have been explored in the context of OC. An in-depth study of the regulation of circRNAs would provide a new theory for the development and progression of OC.

There are abundant unexplored circRNAs related to $\mathrm{OC}$, and circ-FAM53B is a newly discovered circRNA addressed in the present study.circ-FAM53B, also known as hsa_circ_0000267, is located on chr 10: 126370175-126370948, having a spliced sequence length of $773 \mathrm{bp}$. Previously, it was identified as an upregulated circRNA in hepatocellular carcinoma that contributes to oncogenesis by sponging miR-646 (19). In our study, the expression of circ-FAM53B in OC was evaluated, and upregulation of circ-FAM53B was identified in OC specimens and cells. In addition, a survival study demonstrated that the overall survival of patients with high circ-FAM53B expression was significantly lower than that of patients with low circ-FAM53B expression. Moreover, the regulatory effects of circ-FAM53B/miRNA-646/vesicle-associated membrane 
protein 2 (VAMP2) and circ-FAM53B/miRNA-647/mouse double minute 2 (MDM2) on inducing the progression of OC were investigated. These findings may provide new clues for OC treatment.

\section{Materials and methods}

Experimental tissues. Fifty-four pairs of OC tissues/non-tumor tissues were harvested from patients attending the Department of Gynecology, Heilongjiang Provincial Hospital, from November 2012 to June 2014. The mean age of the 54 OC patients included in the study was 51.2. None of the patients received intervention before surgery. An informed consent form was signed by each patient. Approval from the Ethics Committee of Heilongjiang Provincial Hospital was obtained to conduct the present study.

Cell lines and culture. OC cells, HO8910, SKOV3, OVCAR3, and A2780 and one normal cell line (IOSE80) were obtained from Type Culture of Chinese Academy of Sciences (Shanghai, China) or preserved in our laboratory. The cells were maintained in a medium (HyClone; GE Healthcare Life Sciences) containing $10 \%$ fetal bovine serum (FBS; Gibco; Thermo Fisher Scientific. Inc.) in a $37^{\circ} \mathrm{C}, 5 \% \mathrm{CO}_{2}$ incubator, and the medium was replaced every 2-3 days.

RNA extraction and quantification. Total RNA was extracted using TRIzol (Invitrogen; Thermo Fisher Scientific, Inc.) and quantified. The extracted RNA was reverse-transcribed to cDNA. Next, cDNA was amplified using the SYBR Premix Ex Taq II kit (Takara Biotechnology Co., Ltd.). Reaction conditions were as follows: $95^{\circ} \mathrm{C} / 5 \mathrm{~min}, 95^{\circ} \mathrm{C} / 15 \mathrm{sec}, 60^{\circ} \mathrm{C} / 30 \mathrm{sec}$, for 40 cycles. The primers for circ-FAM53B and GAPDH were as follows: circ-FAM53B forward, 5'-ACGACAAGA AGGTCGGTGTT-3' and reverse, 5'-ATTCCCAGATGCTGG TGCTC-3'; GAPDH forward, 5'-GGGAGCCAAAAGGGT CAT-3' and reverse, 5'-GAGTCCTTCCACGATACCAA-3'. The $2^{-\Delta \Delta C q}$ method was applied to quantify the relative gene expression (20).

Cell transfection. Cells were transfected with siRNA-circ-FAM53B/VAMP2/MDM2, vector-circFAM53B/VAMP2/MDM2, and miRNA-646/647 mimics and inhibitors using Lipofectamine 3000 (Invitrogen; Thermo Fisher Scientific, Inc.). The siRNAs specifically targeting the back-spliced junction site of circ-FAM53B were obtained from Shanghai GenePharma Co., Ltd. The targeted sequences were as follows: si-circ-FAM53B-1, 5'-CCAAGATGGCACAGA AAATGA-3'; si-circ-FAM53B-2, 5'-GGCACAGAAAATGAC AGATG-3'; miR-646 mimics, 5'-AAGCAGCUGCCUCUG AGGC-3' (sense), and 5'-CUCAGAGGCAGCUGCUUUU-3' (antisense); miR-646 inhibitor, 5'-GCCUCAGAGGCAGCU GCUU-3'; miR-647 mimics, 5'-GUGGCUGCACUCACU UCCUUC-3' (sense), and 5'-AGGAAGUGAGUGCAGCCA CUU-3' (antisense); and miR-647 inhibitor: GAAGGAAGU GAGUGCAGCCAC.

Cell Counting Kit-8 (CCK-8). Cells were placed in a 96-well plate at a concentration of $2 \times 10^{3}$ cells/well. At the selected time-points $(0,24,48,72$ and $96 \mathrm{~h}), 10 \mu \mathrm{l}$ of reagent (Dojindo
Molecular Technologies, Inc.) was added to each well. After $2 \mathrm{~h}$, the OD value at $450 \mathrm{~nm}$ was read by a microplate reader (Tecan Group, Ltd.), and cell viability curves were plotted.

Cell apoptosis assay. Cells at logarithmic growth phase were digested and centrifuged at $188 \times \mathrm{g}$ for $5 \mathrm{~min}$. The cells were resuspended in $400 \mu \mathrm{l}$ of binding buffer, then stained with $5 \mu \mathrm{l}$ of Annexin V-FITC and $5 \mu \mathrm{l}$ of PI (Beyotime Institute of Biotechnology). A flow cytometer (CyFlow ${ }^{\circledR}$ Cube 6; Sysmex Partec $\mathrm{GmbH}$ ) was utilized to assess cell apoptosis.

Migration and invasion assays. For the wound healing assay, $5 \times 10^{5}$ cells were added to each well and incubated overnight. Cells were detached from the wells using the tip of a $20 \mu \mathrm{l}$ pipette and incubated in serum-free medium for $36 \mathrm{~h}$ (A2780 cells) and $30 \mathrm{~h}$ (HO8910 cells). A light microscope (Leica Microsystems $\mathrm{GmbH}$ ) was used to photograph the wound closure area.

For the Transwell assay, the cells were resuspended in serum-free medium and seeded in the upper unit of Matrigel-coated (BD Biosciences) Transwell unit ( $8 \mu \mathrm{m}$; EMD Millipore), whereas $600 \mu \mathrm{l}$ of complete medium were added into the lower chamber. After $48 \mathrm{~h}$ of incubation, the invasive cells were fixed in $4 \%$ paraformaldehyde (Beijing Solarbio Science \& Technology Co., Ltd.) for $20 \mathrm{~min}$, crystal violet (Beyotime Institute of Biotechnology) staining was performed for $20 \mathrm{~min}$, and the cells were counted using a microscope (Leica Microsystems $\mathrm{GmbH}$ ). A Transwell migration assay was carried out using the same protocol except for the Matrigel pre-coating.

Western blot assay. Total protein was isolated by RIPA buffer (Beyotime Institute of Biotechnology, Beijing, China). The extracted proteins were quantified with a bicinchoninic acid (BCA) Protein Assay Reagent Kit (Beyotime Institute of Biotechnology). In brief, $\mathrm{BCA}$ reagent $\mathrm{A}$ and reagent $\mathrm{B}$ were mixed in a ratio of 50:1 (v/v). Then, $200 \mu \mathrm{l}$ of BCA working solution was added to each well and incubated at $37^{\circ} \mathrm{C}$ for $\sim 30 \mathrm{~min}$. The OD value of each well at $562 \mathrm{~nm}$ was detected using a microplate reader (Tecan Group, Ltd.), and the results were calculated employing a standard curve, plotted using an appropriate standard. Proteins $(40 \mu \mathrm{g})$ were subjected to $10 \%$ SDS-PAGE and transferred to polyvinylidene difluoride (PVDF) membranes (Merck KGaA). The membranes were blocked with $5 \%$ skim milk diluted in Tris-buffered saline containing $0.05 \%$ Tween-20 for $2 \mathrm{~h}$ at room temperature. The membranes were then incubated with primary antibodies against VAMP2 (dilution 1:10,000; cat. no. ab181869), MDM2 (dilution 1:1,000; cat. no. ab38618) or GAPDH (dilution 1:10,000; cat. no. ab181602) (Abcam, Cambridge, MA, USA) at $4^{\circ} \mathrm{C}$ overnight. Next, the blots were incubated in secondary antibody for $2 \mathrm{~h}$ at room temperature (dilution 1:5,000; cat. no. SE134; Solarbio, Beijing, China). The protein bands were obtained using a BeyoECL Plus Kit (Beyotime Institute of Biotechnology) and detected using a chemiluminescence system (Tanon Science and Technology Co., Ltd.). Protein expression levels were normalized to GAPDH. ImageJ 1.48 (National Institutes of Health, Bethesda, MD, USA) software was used to determine the gray value of the protein band. 

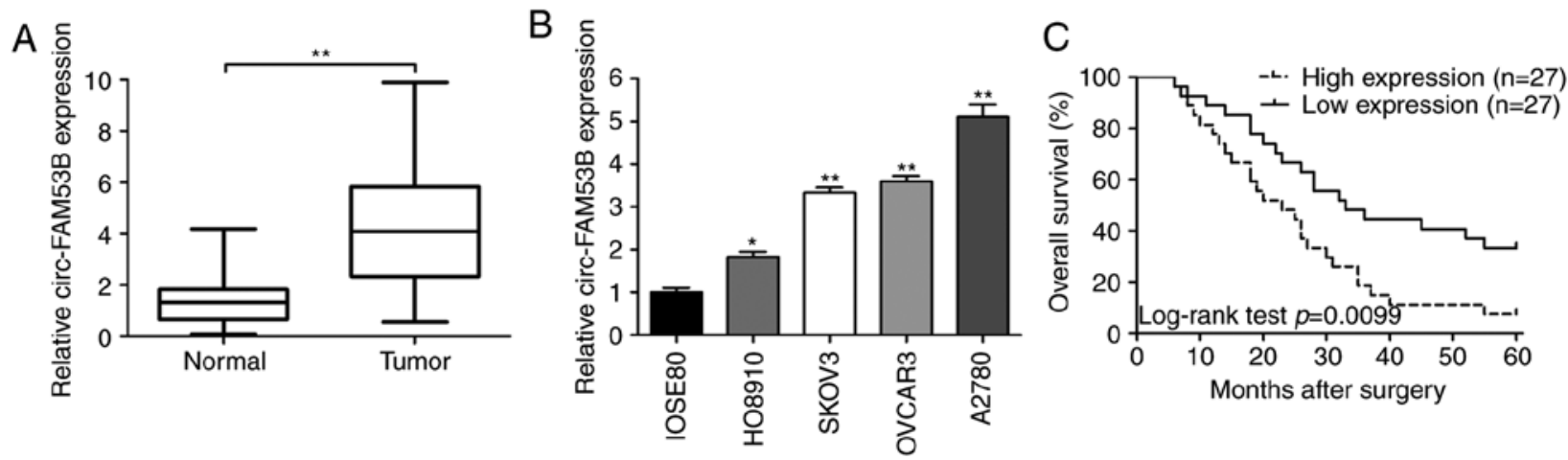

Figure 1. Relative expression of circ-FAM53B in OC tissues and cell lines and its clinical significance. (A) Relative expression of circ-FAM53B in OC tissue samples and their paired non-cancerous tissue samples estimated by qRT-PCR. (B) Relative expression of circ-FAM53B in OC cell lines and normal cell lines assessed by qRT-PCR. (C) Kaplan-Meier survival analysis was used to assess circ-FAM53B expression and the overall survival of OC patients. The data are presented as the mean $\pm \mathrm{SD}(\mathrm{n}=3) .{ }^{*} \mathrm{P}<0.05$ and $^{* *} \mathrm{P}<0.01$. OC, ovarian cancer.

Subcellular fractionation. To assess the cellular localization of circ-FAM53B, cytosolic and nuclear fractions were harvested employing a Nuclear/Cytoplasmic Isolation Kit (Life Technologies; Thermo Fisher Scientific, Inc.). In brief, the cells were lysed and centrifuged $(16,000 \mathrm{x} \mathrm{g})$ at $4^{\circ} \mathrm{C}$, and the supernatant was utilized as a cytosolic fraction. Then, the pellets were washed and incubated with buffer at $4^{\circ} \mathrm{C}$ for $10 \mathrm{~min}$ and used as a nuclear fraction.

Target prediction and dual luciferase reporter gene assay. The possibility of interaction between circ-FAM53B and miR-646/miR-647 was predicted using the Circular RNA Interactome online database (https://circinteractome.nia.nih.gov). The downstream targets of miR-646 and miR-647 were analyzed by TargetScan Human 7.2 database (http://www.targetscan. org/vert_72/). To verify these predicted interactions, a dual luciferase reporter gene assay was carried out. circFAM53B/VAMP2/MDM2 Wt and circ-FAM53B/VAMP2/ MDM2 Mut were constructed based on the predicted binding sequences to miRNA-646 and miRNA-647. Cells were co-transfected with circ-FAM53B/VAMP2/MDM2 Wt or circ-FAM53B/VAMP2/MDM2 Mut and miRNA-646-NC or miRNA-646 mimic for $48 \mathrm{~h}$. Cells were also co-transfected with circ-FAM53B/VAMP2/MDM2 Wt or circ-FAM53B/VAMP2/MDM2 Mut and miRNA-647-NC or miRNA-647 mimic for $48 \mathrm{~h}$. After cell lysis, relative luciferase activity was determined (Promega Corp.).

Data analysis. The data are displayed as the mean \pm standard deviation (SD) based on three independent experiments. Statistical analyses were carried out using GraphPad Prism 5.01 software (GraphPad, Inc.). The differences between groups were analyzed by Student's $t$-test or one-way ANOVA (followed by Tukey's multiple comparison test). Correlation between the circ-FAM53B level and pathological indexes of OC patients was analyzed through the Fisher's exact test. The Kaplan-Meier methods were applied to analyze the survival probability of OC patients. Valuation was performed using the log-rank test. Cox regression analysis was utilized to explore the independent prognostic factors for the patients with OC. A P-value $<0.05$ was considered to indicate a statistically significant difference.

\section{Results}

Overexpression of circ-FAM53B is identified in OC tissue samples and cell lines and is associated with adverse prognosis. The expression of circ-FAM53B in 54 cases of OC tissues and paired noncancerous samples was assessed by quantitative real-time polymerase chain reaction (qRT-PCR). As displayed in Fig. 1A, circ-FAM53B was upregulated in OC tissues compared to that in their counterparts. Similarly, upregulation of circ-FAM53B was revealed in OC cell lines, including HO8910, SKOV3, OVCAR3, and A2780, compared to that in IOSE80 cells (Fig. 1B). As depicted in Table I, the circ-FAM53B level was related to tumor size $(\mathrm{P}=0.028)$, FIGO stages $(\mathrm{P}=0.013)$, and lymph node invasion $(\mathrm{P}=0.006)$ of $\mathrm{OC}$ (Table I). Moreover, Kaplan-Meier curves revealed a poor survival rate in OC patients with upregulated circ-FAM53B (Fig. 1C). Univariate analysis indicated FIGO stage $(\mathrm{P}=0.049)$ and circ-FAM53B expression $(\mathrm{P}=0.013)$ as two factors related to adverse prognosis of patients. Further multivariate analysis demonstrated that circ-FAM53B could be regarded as an independent prognostic indicator $(\mathrm{P}=0.049$; Table II).

circ-FAM53B plays an oncogenic role in OC cells. To verify the oncogenic role of circ-FAM53B in OC progression, functional assays were conducted. We harvested optimally transfected cells after $48 \mathrm{~h}$ and detected the relative expression of circ-FAM53B. The high circ-FAM53B expression in the A2780 cell line was decreased by transfection with si-circ-FAM53B-1 or si-circ-FAM53B-2 (Fig. 2A). si-circ-FAM53B-1 had a more effective knockdown efficiency than si-circ-FAM53B-2; thus, it was selected for further assays. Plasmid transfection technology was applied using a low-expression circ-FAM53B cell line, HO8910. As revealed in Fig. 2B, the overexpression efficiency was satisfactory. Cell viability in the si-circ-FAM53B-1 group was significantly decreased in the A2780 cell line (Fig. 2C), whereas overexpression of circ-FAM53B enhanced the viability of HO8910 cells (Fig. 2C). Flow cytometric analysis also demonstrated that the low expression of circ-FAM53B markedly increased the apoptosis rate of A2780 cells (Fig. 2D). In addition, ectopically expressed circ-FAM53B significantly decreased HO8910 cell apoptosis (Fig. 2D). 
Table I. circ-FAM53B expression and clinicopathologic characteristics of OC patients.

\begin{tabular}{|c|c|c|c|c|}
\hline \multirow[b]{2}{*}{ Clinicopathologic variables } & \multirow[b]{2}{*}{ No. of patients } & \multicolumn{2}{|c|}{ Circ-FAM53B expression } & \multirow[b]{2}{*}{ P-value } \\
\hline & & $\operatorname{High}(\%)$ & Low $(\%)$ & \\
\hline Age (years) & & & & 0.158 \\
\hline$<50$ & 34 & $14(25.93)$ & $20(37.04)$ & \\
\hline$\geq 50$ & 20 & $13(24.07)$ & $7(12.96)$ & \\
\hline Tumor size $(\mathrm{cm})$ & & & & 0.028 \\
\hline$<3$ & 29 & $10(18.52)$ & $19(35.19)$ & \\
\hline$\geq 3$ & 25 & $17(31.48)$ & $8(14.81)$ & \\
\hline Histology & & & & 0.773 \\
\hline Serous & 36 & $19(35.19)$ & $17(31.48)$ & \\
\hline Mucinous & 18 & $8(14.81)$ & $10(18.52)$ & \\
\hline FIGO stage & & & & 0.013 \\
\hline I-II & 24 & $7(12.96)$ & $17(31.48)$ & \\
\hline III-IV & 30 & $20(37.04)$ & $10(18.52)$ & \\
\hline Lymph node metastasis & & & & 0.006 \\
\hline Negative & 29 & $9(16.67)$ & $20(37.04)$ & \\
\hline Positive & 25 & $18(33.33)$ & $7(12.96)$ & \\
\hline Differentiation grade & & & & 0.577 \\
\hline Well/Moderately & 33 & $15(27.78)$ & $18(33.33)$ & \\
\hline Poorly & 21 & $12(22.22)$ & $9(16.67)$ & \\
\hline
\end{tabular}

Table II. Univariate and multivariate analysis of prognostic factors for overall survival in OC patients.

\begin{tabular}{|c|c|c|c|c|c|c|}
\hline \multirow[b]{2}{*}{ Variables } & \multicolumn{3}{|c|}{ Univariate analysis } & \multicolumn{3}{|c|}{ Multivariate analysis } \\
\hline & HR & $95 \% \mathrm{CI}$ & P-value & HR & $95 \% \mathrm{CI}$ & P-value \\
\hline \multicolumn{7}{|l|}{ Overall survival } \\
\hline Age $(\geq 50$ vs. $<50)$ & 0.978 & $0.527-1.816$ & 0.944 & & & \\
\hline Tumor size ( $\geq 3 \mathrm{~cm}$ vs. $<3 \mathrm{~cm})$ & 1.319 & $0.724-2.403$ & 0.366 & & & \\
\hline Histology (serous vs. mucinous) & 1.307 & $0.681-2.507$ & 0.420 & & & \\
\hline FIGO stage (III-IV vs. I-II) & 1.857 & $1.003-3.438$ & 0.049 & & & 0.218 \\
\hline Lymph node metastasis (positive vs. negative) & 1.700 & $0.930-3.106$ & 0.085 & & & \\
\hline Differentiation grade (poorly vs. well/moderately) & 1.179 & $0.633-2.196$ & 0.604 & & & \\
\hline circ-FAM53B expression (high vs. low) & 2.187 & $1.181-4.050$ & 0.013 & 1.915 & $1.002-3.666$ & 0.049 \\
\hline
\end{tabular}

OC, ovarian cancer; HR, hazard ratio; 95\% CI, 95\% confidence interval.

Downregulation of circ-FAM53B expression decreased the wound closure area in A2780 cells (Fig. 2E), whereas upregulation of circ-FAM53B increased the HO8910 cell migratory potential (Fig. 2E). Moreover, downregulation of circ-FAM53B expression could decrease the migratory and invasive rates in the A2780 cell line (Fig. 2F). An increased level of circ-FAM53B enhanced metastatic properties in HO8910 cells (Fig. 2F). These data indicated that high levels of circ-FAM53B contribute to cell progression in OC cell lines.
circ-FAM53B sponges miR-646 and miR-647 to increase $V A M P 2$ and MDM2 expression, respectively. To explore the mechanisms of action of circ-FAM53B in OC cells, the localization of circ-FAM53B in OC cells was first analyzed by a subcellular fractionation assay. The results indicated that circ-FAM53B was enriched in the cytoplasm (Fig. 3A), indicating that circ-FAM53B is capable of modulating gene expression at the post-transcriptional level. Thus, the miRNAs that may interact with circ-FAM53B were further predicted, using the Circular RNA Interactome online database. From 

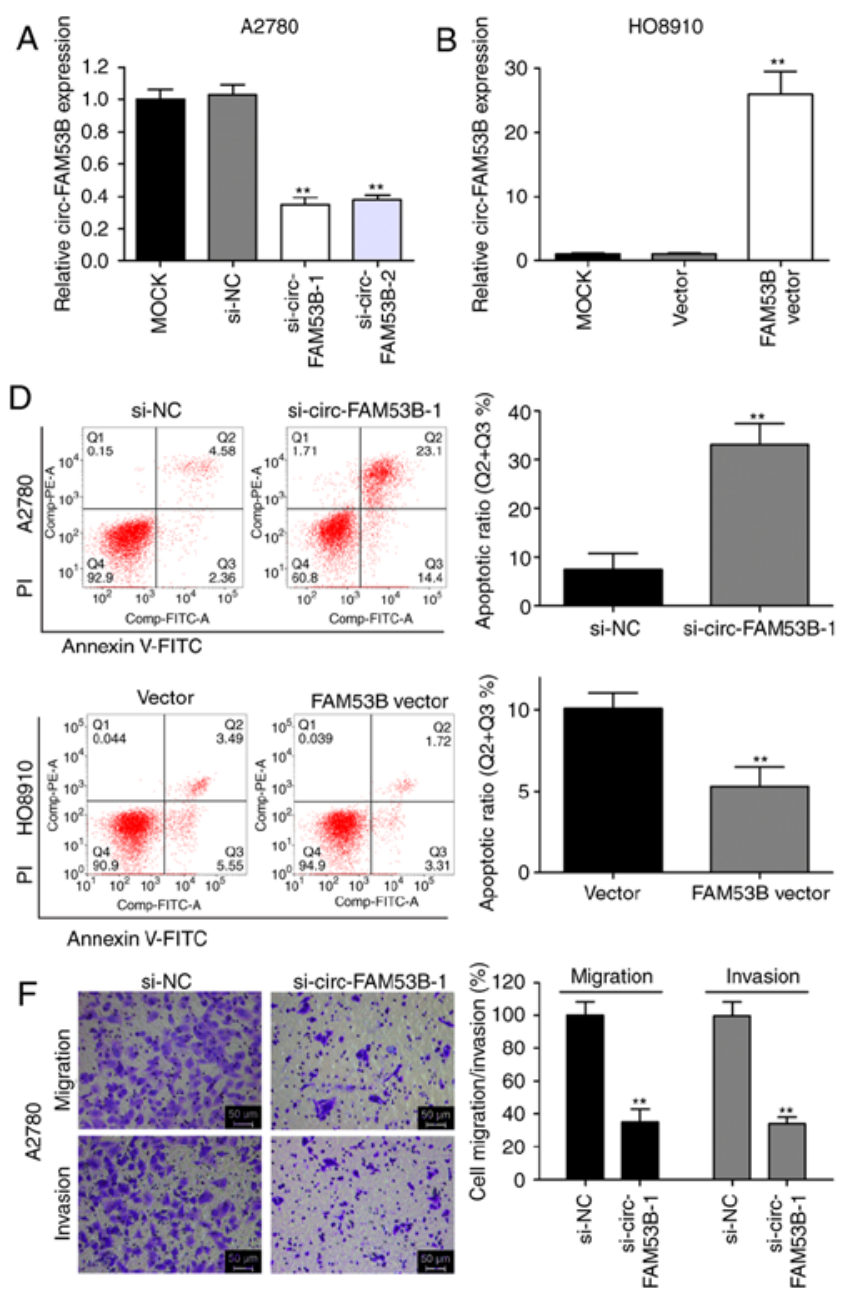

C
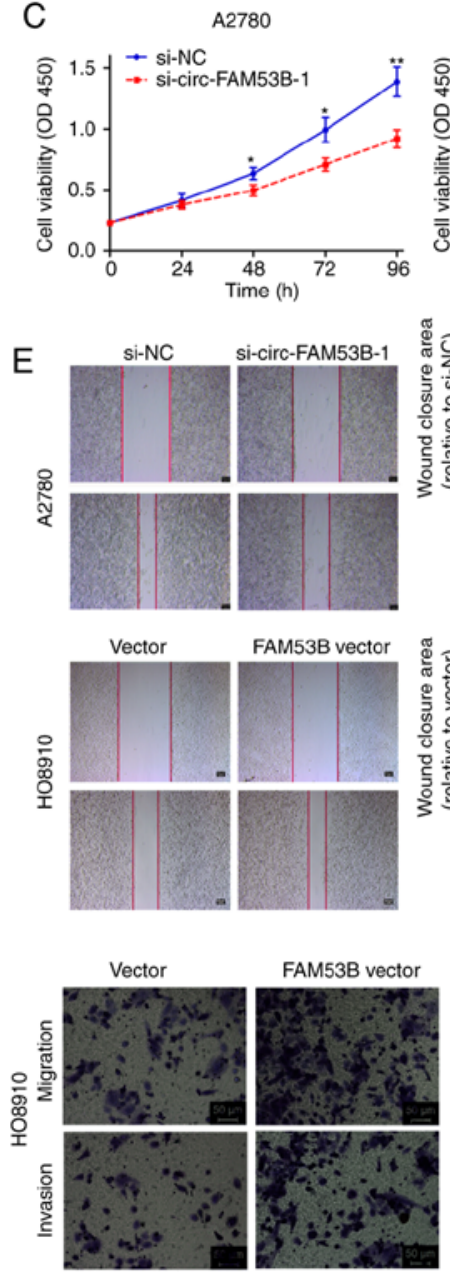
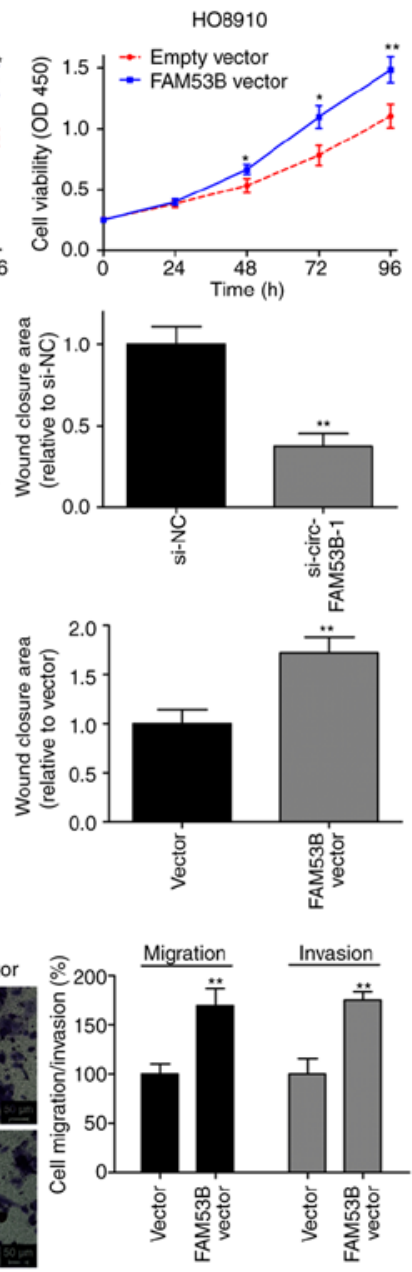

Figure 2. circ-FAM53B promotes OC cell progression. (A and B) circ-FAM53B expression was detected after transfection in (A) A2780 cells and in (B) HO8910 cells by qRT-PCR. (C) CCK-8 assays were used to detect viability of A2780 and HO8910 cells after transfection. (D) Flow cytometric analysis was used to detect apoptosis of A2780 and HO8910 cells after transfection. (E) Wound healing assays were used to detect cell migration of A2780 and HO8910 cells after transfection. (F) Transwell assays were used to detect cell migration and invasion capacities of A2780 and HO8910 cells after transfection. Data are presented as the mean $\pm \mathrm{SD}(\mathrm{n}=3)$. ${ }^{*} \mathrm{P}<0.05$ and ${ }^{* *} \mathrm{P}<0.01$. OC, ovarian cancer.

the seven predicted miRNAs (miR-31, miR-144, miR-198, miR-503, miR-532, miR-646, and miR-647), it was revealed that the expression of miR-646 and miR-647 were modulated by circ-FAM53B levels in A2780 and HO8910 cell lines (Fig. 3B). A negative correlation between circ-FAM53B and miR-646/miR-647 was also identified in the OC tissue samples (Fig. 3C and D). In addition, miR-646 and miR-647 were predicted to bind to the sequence of circ-FAM53B. Overexpression of miR-646 decreased the luciferase intensity of circ-FAM53B Wt, indicating its binding condition (Fig. 3E and F). Similarly, miR-647 could bind to the seed sequence of circ-FAM53B, as verified by the luciferase reporter gene assay (Fig. 3E and G). Therefore, it was concluded that circ-FAM53B sponges miR-646 and miR-647 in OC cells. Subsequently, the downstream targets of miR-646 and miR-647 with an oncogenic role were predicted and analyzed by TargetScan Human 7.2 database. miR-646 and miR-647 were predicted to have a potential to interact with VAMP2 3'-UTR and MDM2 3'-UTR, respectively. As revealed in Fig. 4A and B, miR-646 and miR-647 mimics could significantly enhance the expression levels of miR-646 and miR-647, respectively. In addition, qRT-PCR data revealed that miR-646 and miR-647 inhibitor significantly downregulated miR-646 and miR-647 expression, respectively. Transfection with miR-646 or miR-647 mimics could significantly downregulate the expression of VAMP2 and MDM2, respectively (Fig. 4C). In addition, transfection with miR-646 or miR-647 inhibitor enhanced VAMP2 and MDM2 expression, respectively (Fig. 4C). Moreover, it was revealed that circ-FAM53B expression was positively correlated with the levels of VAMP2 and MDM2 in OC tissues (Fig. 4D and E). Furthermore, the dual-luciferase reporter gene assay confirmed an interaction between miR-646 and VAMP2 3'-UTR (Fig. 4F and G). A similar result was also revealed with miR-647 and MDM2 3'-UTR (Fig. 4F and H). These data indicated that VAMP2 and MDM2 are the downstream targets of miR-646 and miR-647, respectively. The expression of VAMP2 and MDM2 was decreased after the silencing of circ-FAM53B, while co-transfection with inh-miR-646/VAMP2 vector or inh-miR-647/MDM2 vector partially restored VAMP2 and MDM2 expression, respectively. Additionally, miR-646 mimics or si-VAMP2 could partially inhibit the high VAMP2 expression revealed by the circ-FAM53B vector. Co-transfection with either miR-647 mimics or si-MDM2 


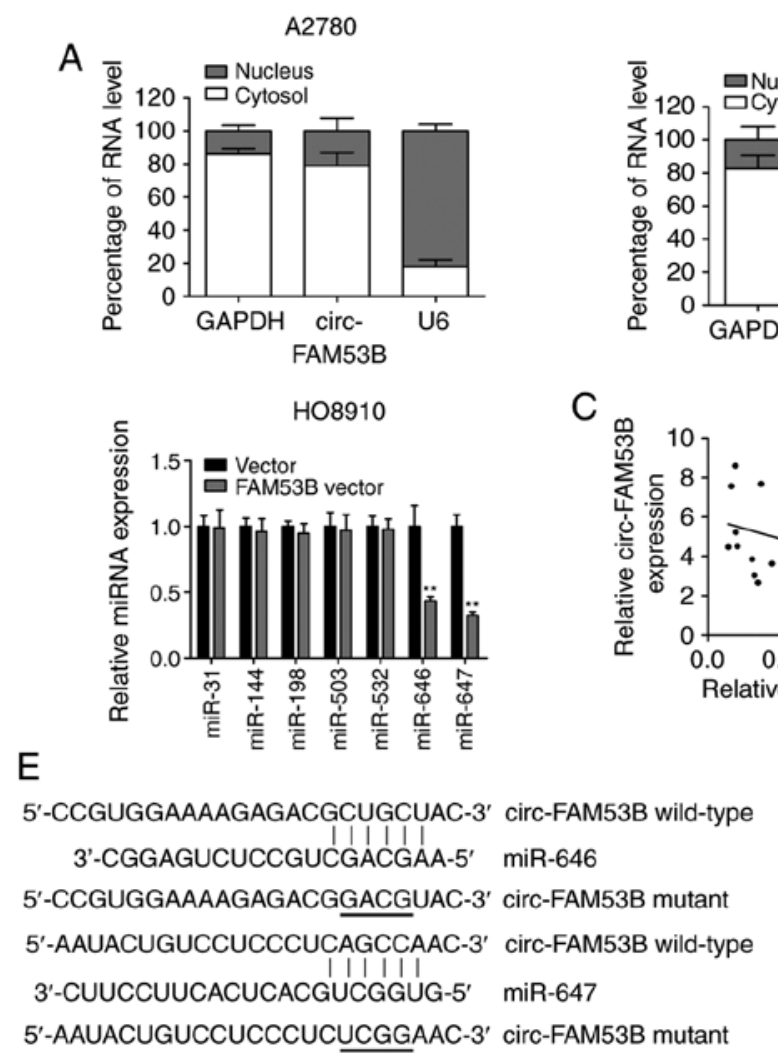

H08910

cleus

Cytosol
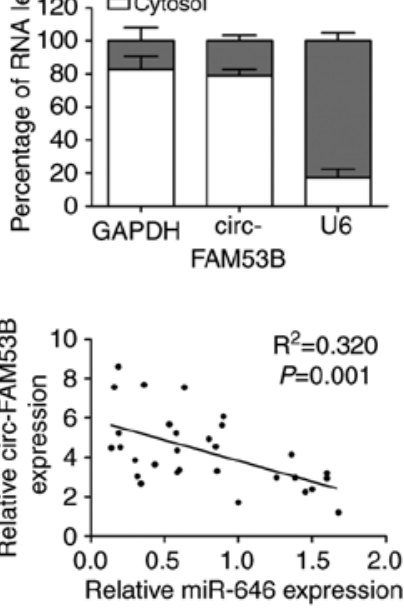

B

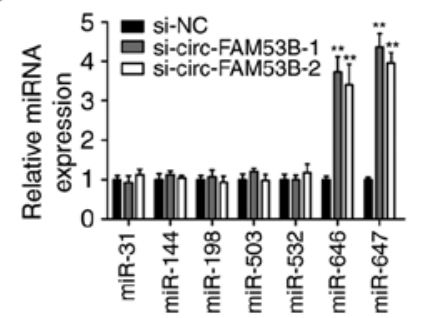

D

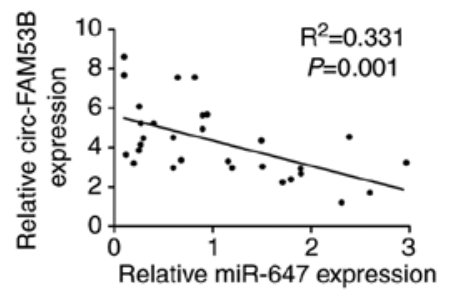

Figure 3. circ-FAM53B sponges miR-646 and miR-647. (A) Detection of the percentage of circ-FAM53B, GAPDH and U6 in the cytoplasm and nuclear fractions of A2780 and HO8910 cells using qRT-PCR. GAPDH and U6 were used as cytoplasmic and nuclear localization markers, respectively. (B) Relative miRNA expression was detected in A2780 and HO8910 cells after transfection. (C) Correlation analysis of circ-FAM53B and miR-646 in the tissues of OC patients. (D) Correlation analysis of circ-FAM53B and miR-647 in the tissues of OC patients. (E) Diagrammatic sketch of the binding sites for circ-FAM53B and miR-646/miR-647. (F) A luciferase reporter assay was conducted to evaluate the interactive ability between miR-646 and circ-FAM53B. (G) A luciferase reporter assay was conducted to evaluate the interactive ability between miR-647 and circ-FAM53B. (A-D and F and G) Data are presented as the mean \pm SD $(\mathrm{n}=3) .{ }^{*} \mathrm{P}<0.05$ and $^{* *} \mathrm{P}<0.01$. OC, ovarian cancer.

partially decreased the high MDM2 expression induced by the circ-FAM53B vector (Fig. 4I).

The oncogenic functions of circ-FAM53B are partially attributed to its modulation of VAMP2 and MDM2. In the present experiment, circ-FAM53B silencing attenuated the viability of A2780 cells, which was partly reversed after co-transfection with VAMP2 or MDM2 vector (Fig. 5A). In HO8910 cells transfected with circ-FAM53B vector, cell viability was significantly increased, which was reversed by transfection with si-VAMP2 or si-MDM2 (Fig. 5A). Similar results were also observed in the flow cytometric experiment (Fig. 5B). The inhibited invasive capacity of A2780 cells with circ-FAM53B knockdown was partially reversed by VAMP2 or MDM2 vector (Fig. 5C). Similarly, the increased invasion of HO8910 cells with overexpressed circ-FAM53B was reversed by si-VAMP2 and si-MDM2 (Fig. 5C).

\section{Discussion}

$\mathrm{OC}$ is developing into a serious disease, thereby posing a great burden on human health. RNA sequencing provides a new strategy for the treatment of human cancers, including OC $(18,21,22)$. circ-FAM53B is located on chr 10: $126370175-126370948$, and its spliced length is $773 \mathrm{bp}$. It was identified as an upregulated circRNA in hepatocellular carcinoma, contributing to oncogenesis by sponging miR-646 (19). However, its expression pattern, clinical significance, functions, and molecular mechanisms in other cancers remain unclear. In the present study, circ-FAM53B was revealed to be elevated in $\mathrm{OC}$ and was closely related to clinical severity of OC patients. Consequently, it can be used as an independent prognostic predictor for $\mathrm{OC}$ patients. Overexpression of circ-FAM53B accelerated OC cells to proliferate, migrate, and invade the ovaries, whereas silencing of circ-FAM53B caused the opposite effect. Furthermore, circ-FAM53B was mainly localized in the cytoplasm, which indicated its mechanism of gene regulation at the post-transcriptional level.

miRNAs are also identified as a class of noncoding RNAs $(23,24)$. A growing number of studies have revealed that miRNAs play a significant role in regulating gene expression patterns during physiological and cell biological processes (25). Some identified miRNAs can be recognized as effectors of oncogenesis. For example, miR-21 is an anti-apoptotic factor in gliomas (26). A majority of animal microRNAs, which have been characterized thus far, affect protein synthesis of their target mRNAs (25). A hypothesis related to competing endogenous RNA (ceRNA) has been recently proposed, and various studies have reported this mechanism in the field of RNA research. For example, hsa_circ_0076248 can promote the progression of glioma by affecting the miR-181a/SIRT1 pathway (27). Therefore, circRNAs and miRNAs and the correlation between 


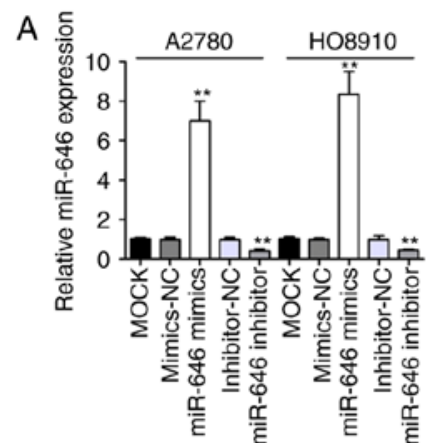

D

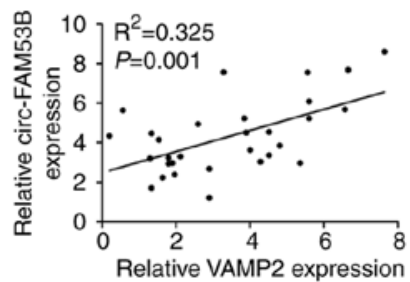

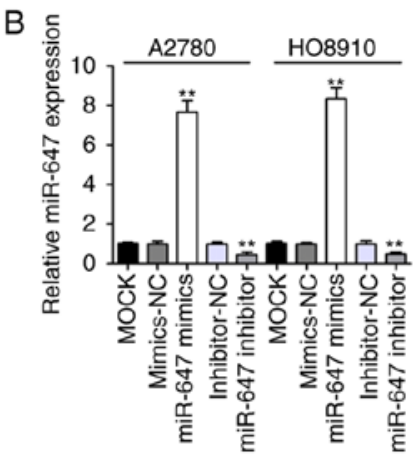

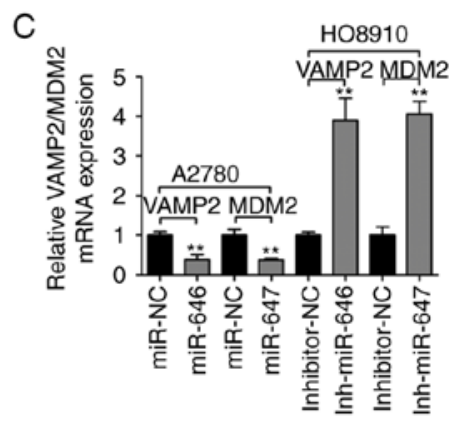

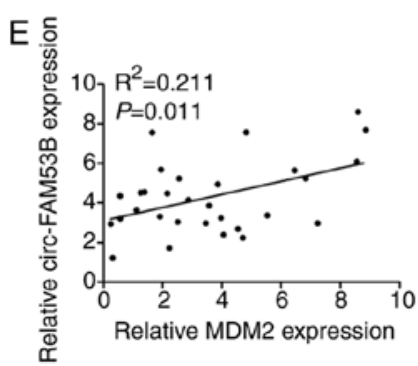
F 5'-GCCAAAAACACAUAUAGCUGCUA-3'
1111111
3'-CGGAGUCUCCGUCGACGAA-5'
5'-GCCAAAAACACAUAUACGACGUA-3'
3'GUGAGCCACCAUGUCCAGCCAAG-5'
I11 III
5'-CUUCCUUCACUCACGUCGGUG-3'
5'-GUGAGCCACCAUGUCCUCGGAAG-3'

VAMP2 3' UTR wild-type miR-646

VAMP2 3' UTR mutant

MDM2 3' UTR wild-type

$\operatorname{miR}-647$

MDM2 3' UTR mutant
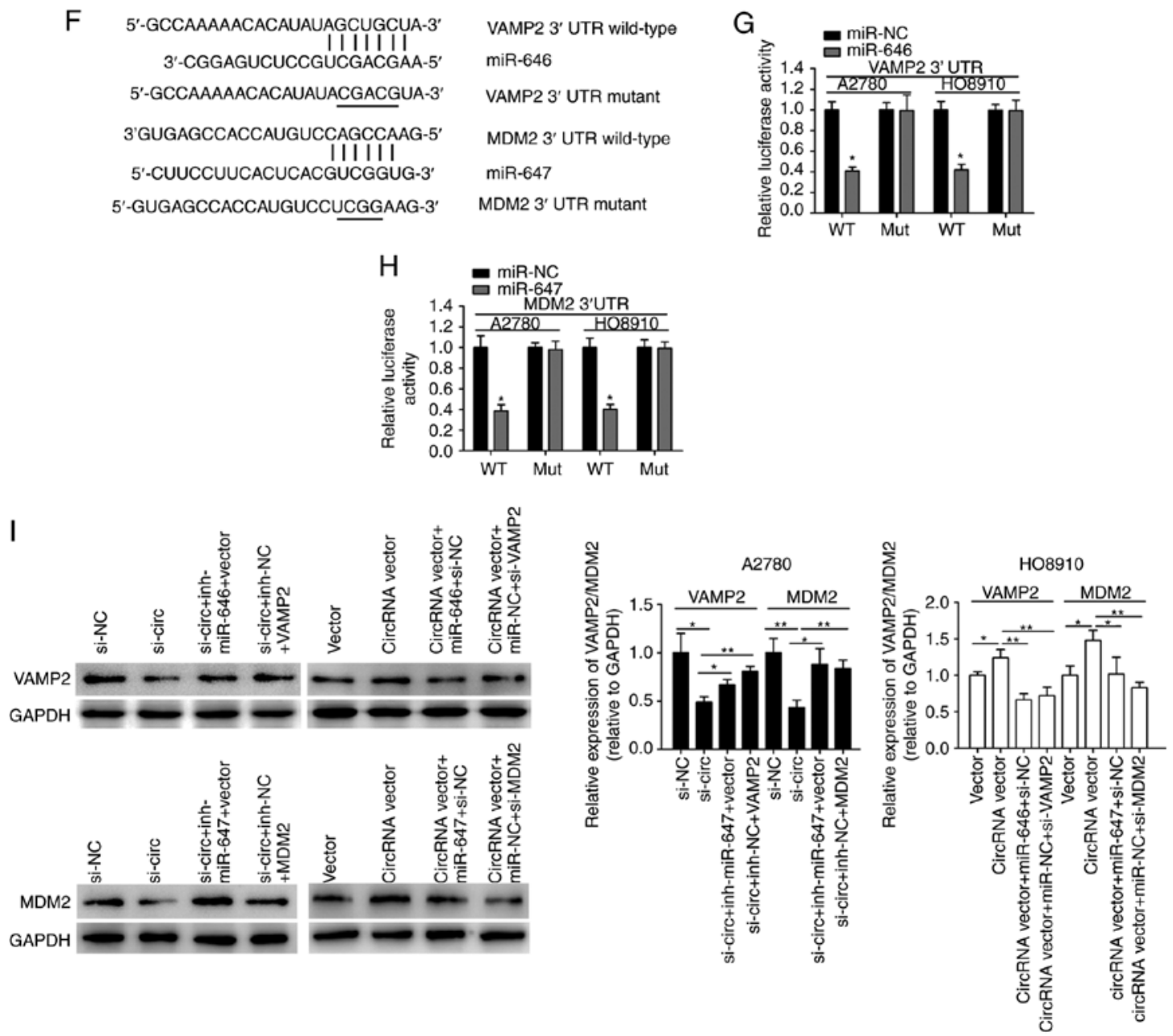

Figure 4. circ-FAM53B sponges miR-646 and miR-647 to increase VAMP2 and MDM2 expression, respectively. (A) Relative expression of miR-646 was detected in A2780 and HO8910 cells after transfection. miR-646 mimics were compared with mimics-NC. miR-646 inhibitor was compared with inhibitor-NC. (B) Relative expression of miR-647 was detected in A2780 and HO8910 cells after transfection. miR-647 mimics were compared with mimics-NC. miR-647 inhibitor was compared with inhibitor-NC. (C) Relative VAMP2 and MDM2 expression was detected in A2780 and HO8910 cells after transfection. (D) Correlation analysis of circ-FAM53B and VAMP2 mRNA in the tissues of OC patients. (E) Correlation analysis of circ-FAM53B and MDM2 mRNA in the tissues of OC patients. (F) Diagrammatic sketch of the binding sites for 3'-UTR of VAMP2/MDM2 and miR-646/miR-647. (G) A luciferase reporter assay was conducted to evaluate the interaction ability between VAMP2 3'-UTR and miR-646. (H) A luciferase reporter assay was conducted to evaluate the interaction ability between MDM2 3'-UTR and miR-647. (I) VAMP2 and MDM2 expression was detected in A2780 and HO8910 cells after transfection. (A-C and G-I) Data are presented as the mean $\pm \mathrm{SD}(\mathrm{n}=3) .{ }^{*} \mathrm{P}<0.05,{ }^{* *} \mathrm{P}<0.01$. OC, ovarian cancer. 
A
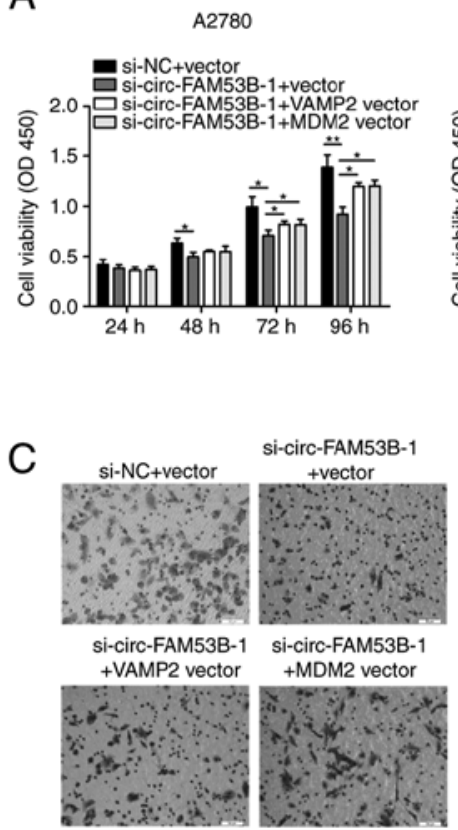

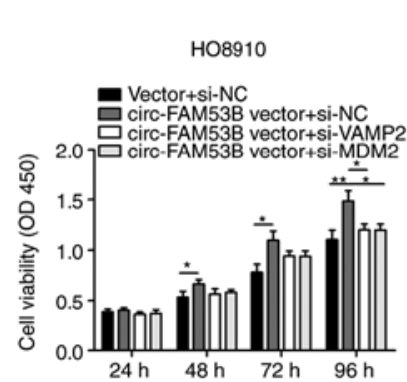

B

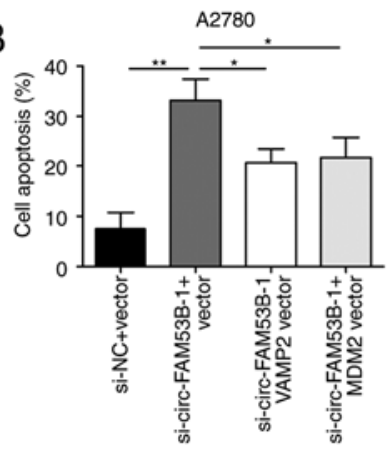

A2780
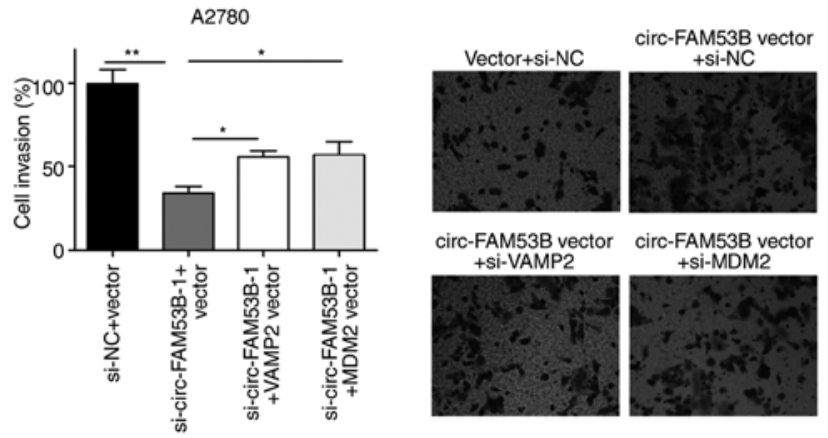
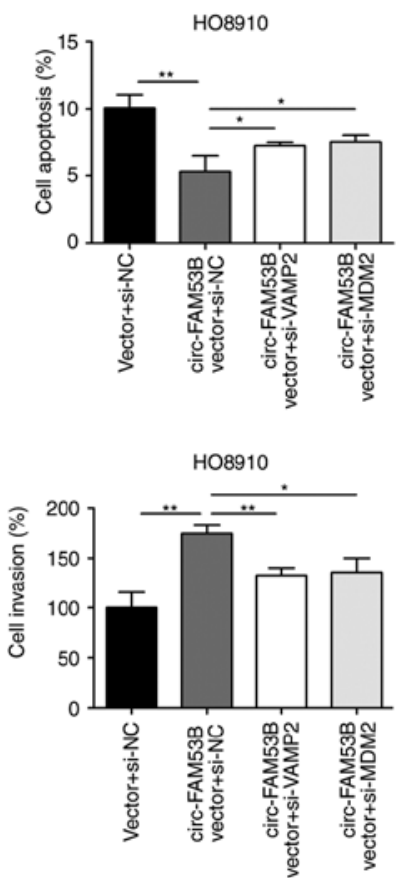

Figure 5. Oncogenic role of circ-FAM53B is dependent on its regulation of VAMP2 and MDM2. (A) A CCK-8 assay was used to evaluate viability after transfection in A2780 and HO8910 cells. (B) Flow cytometric assay was used to evaluate apoptosis after transfection in A2780 and HO8910 cells. (C) Transwell assays were used to evaluate invasive potential after transfection in A2780 and HO8910 cells. (A-C) Data are presented as the mean \pm SD ( $=3$ ). ${ }^{*} \mathrm{P}<0.05$ and ${ }^{* *} \mathrm{P}<0.01$. OC, ovarian cancer.

them should be considered as a significant measure to study OC. Several studies have demonstrated that circRNAs interact with target miRNAs and suppress the downstream targets, thus regulating the behaviors of tumor cells (23). For example, circ-ERBB2 can promote gastric cancer development and progression through the regulation of miR-503/CACUL1 and miR-637/MMP-19 signaling (24). Previously, Pan et al revealed an interaction between miR-646 and circ-FAM53B in hepatocellular carcinoma (19). In the present study, miR-646 and miR-647 were identified as two miRNAs which can be sponged by circ-FAM53B in OC cells. miR-646 and miR-647 have been reported to act as tumor suppressors in certain human cancers. It is reported that miR-646 could be sponged by oncogenic lncRNA HOTAIR to mediate estrogen-induced metastasis of endometrial cancer cells (28). Another study revealed that miR-646 exerts a tumor-suppressive role in gastric cancer by modulating FOXK1 (29). In addition, miR-647 was revealed to suppress progression in gastric cancer and NSCLC $(30,31)$. Additionally, SRF-MYH9 and TRAF2 were identified as downstream targets of miR-647 in gastric cancer and NSCLC, respectively $(30,31)$.

In the present study, it was further revealed that VAMP2 and MDM2 are the downstream targets of miR-646 and miR-647, respectively. VAMP2 was first identified in synaptic vesicles from rat brain, and has been revealed to be implicated in synaptic vesicle docking and fusion with plasma membrane proteins (32). In addition, it has been revealed to be significantly upregulated in a wide range of malignancies, including bladder cancer (33), osteosarcoma (34), and other cancers. MDM2 is an oncoprotein that facilitates rapid degradation of the tumor suppressor, p53 (35). In contrast to other tumor suppressors, such as Rb, p16, or PTEN, p53 is frequently overexpressed in tumors, although its function is ablated. Basically, mutant p53 is frequently upregulated in ovarian cancer cells; however, wild-type p53 is diminished in these cells. The function of wild-type p53 is frequently decreased due to different alterations, which result in an enhanced activity of its negative regulators, such as MDM2. Suppression of the interaction between MDM2 and p53 could stabilize the p53 protein, thus leading to suppression of cancer progression (36). Various studies have indicated its oncogenic function in tumorigenesis, including esophageal squamous cell carcinoma (37), breast cancer (38), and lung adenocarcinoma (39). However, the role and mechanism of VAMP2 and MDM2 in OC have not been studied to date. In the present study, circ-FAM53B was identified as a ceRNA to competitively bind to miR-646 and miR-647 to upregulate VAMP2 and MDM2 expression, thus mediating the behaviors of OC cells. OC is a multi-factorial, multi-step, complex disease with many dysregulated circRNAs/miRNAs. This study merely reveals the tip of the iceberg in the development of OC and its progression.

In summary, circ-FAM53B was markedly overexpressed in tissues and cells of $\mathrm{OC}$, and it was associated with clinical severity and adverse prognosis in OC patients. Additionally, circ-FAM53B contributed to OC cell progression by sponging miR-646 and miR-647, thus increasing the expression of VAMP2 and MDM2, respectively.

\section{Acknowledgements}

Not applicable.

\section{Funding}

No funding was received. 


\section{Availability of data and materials}

The datasets supporting the project are available from the corresponding author upon reasonable request.

\section{Authors' contributions}

JL designed the study and provided the final approval of the version to be published. DS and LZ performed the experiments. DS and JL analyzed the data. All authors have approved the final version of the publication and agree to be accountable for all aspects of the research in ensuring that the accuracy or integrity of any part of the work are appropriately investigated and resolved.

\section{Ethics approval and consent to participate}

Approval from the Ethics Committee of Heilongjiang Provincial Hospital was obtained to conduct the present study and an informed consent form was signed by each patient.

\section{Patient consent for publication}

Not applicable.

\section{Competing interests}

The authors declare that they have no competing interests.

\section{References}

1. Bast RC Jr, Hennessy B and Mills GB: The biology of ovarian cancer: New opportunities for translation. Nat Rev Cancer 9: 415-428, 2009.

2. Siegel R, Naishadham D and Jemal A: Cancer statistics, 2013. CA Cancer J Clin 63: 11-30, 2013.

3. Timmermans M, Sonke GS, Slangen BFM, Baalbergen A, Bekkers RLM, Fons G, Gerestein CG, Kruse AJ, Roes EM Zusterzeel PLM, et al: Outcome of surgery in advanced ovarian cancer varies between geographical regions; opportunities for improvement in the netherlands. Eur J Surg Oncol 45: 1425-1431, 2019.

4. Morice P, Gouy S and Leary A: Mucinous ovarian carcinoma. N Engl J Med 380: 1256-1266, 2019.

5. Stewart C, Ralyea C and Lockwood S: Ovarian cancer: An integrated review. Semin Oncol Nurs 35: 151-156, 2019.

6. Memczak S, Jens M, Elefsinioti A, Torti F, Krueger J, Rybak A, Maier L, Mackowiak SD, Gregersen LH, Munschauer M, et al: Circular RNAs are a large class of animal RNAs with regulatory potency. Nature 495: 333-338, 2013.

7. Jeck WR, Sorrentino JA, Wang K, Slevin MK, Burd CE, Liu J, Marzluff WF and Sharpless NE: Circular RNAs are abundant, conserved, and associated with ALU repeats. RNA 19: 141-157, 2013.

8. Zang J, Lu D and Xu A: The interaction of circRNAs and RNA binding proteins: An important part of circRNA maintenance and function. J Neurosci Res Dec 21, 2018 (Epub ahead of print).

9. Hao L, Rong W, Bai L, Cui H, Zhang S, Li Y, Chen D and Meng X: Upregulated circular RNA circ_0007534 indicates an unfavorable prognosis in pancreatic ductal adenocarcinoma and regulates cell proliferation, apoptosis, and invasion by sponging miR-625 and miR-892b. J Cell Biochem 120: 3780-3789, 2019.

10. Xu Y, Yao Y, Zhong X, Leng K, Qin W, Qu L, Cui Y and Jiang X: Downregulated circular RNA hsa_circ_0001649 regulates proliferation, migration and invasion in cholangiocarcinoma cells. Biochem Biophys Res Commun 496: 455-461, 2018.

11. Aufiero S, Reckman YJ, Pinto YM and Creemers EE: Circular RNAs open a new chapter in cardiovascular biology. Nat Rev Cardiol 16: 503-514, 2019.
12. Li KS, Pan F, Mao XD, Liu C and Chen YJ: Biological functions of circular RNAs and their roles in occurrence of reproduction and gynecological diseases. Am J Transl Res 11: 1-15, 2019.

13. Bach DH, Lee SK and Sood AK: Circular RNAs in cancer. Mol Ther Nucleic Acids 16: 118-129, 2019.

14. Yang W, Liu Y, Gao R, Xiu Z and Sun T: Knockdown of cZNF292 suppressed hypoxic human hepatoma SMMC7721 cell proliferation, vasculogenic mimicry, and radioresistance. Cell Signal 60: 122-135, 2019.

15. Matboli M, Shafei AE, Ali MA, Ashry AM, Kamal KM, Agag MA, Reda I, Tash EF and Ali M: circRNAs (hsa_ circ_00156, hsa_circ_000224, and hsa_circ_000520) are novel potential biomarkers in hepatocellular carcinoma. J Cell Biochem: Nov 13, 2018 (Epub ahead of print).

16. Guo J, Duan H, Li Y, Yang L and Yuan L: A novel circular RNA circ-ZNF652 promotes hepatocellular carcinoma metastasis through inducing snail-mediated epithelial-mesenchymal transition by sponging miR-203/miR-502-5p. Biochem Biophys Res Commun 513: 812-819, 2019.

17. Wang T, Wang X, Du Q, Wu N, Liu X, Chen Y and Wang X: The circRNA circP4HB promotes NSCLC aggressiveness and metastasis by sponging miR-133a-5p. Biochem Biophys Res Commun 513: 904-911, 2019.

18. Gao Y, Zhang C, Liu Y and Wang M: Circular RNA profiling reveals circRNA1656 as a novel biomarker in high grade serous ovarian cancer. Biosci Trends 13: 204-211, 2019.

19. Pan H, Tang L, Jiang H, Li X, Wang R, Gao J and Li Q: Enhanced expression of circ_0000267 in hepatocellular carcinoma indicates poor prognosis and facilitates cell progression by sponging miR-646. J Cell Biochem: Feb 5, 2019 (Epub ahead of print).

20. Livak KJ and Schmittgen TD: Analysis of relative gene expression data using real-time quantitative PCR and the 2(-Delta Delta C(T)) method. Methods 25: 402-408, 2001.

21. Yang C, Wei Y, Yu L and Xiao Y: Identification of altered circular RNA expression in serum exosomes from patients with papillary thyroid carcinoma by high-throughput sequencing. Med Sci Monit 25: 2785-2791, 2019.

22. Teng F, Xu J, Zhang M, Liu S, Gu Y, Zhang M, Wang X, Ni J, Qian B, Shen R and Jia X: Comprehensive circular RNA expression profiles and the tumor-suppressive function of circHIPK 3 in ovarian cancer. Int J Biochem Cell Biol 112: 8-17, 2019.

23. Mao W, Huang X, Wang L, Zhang Z, Liu M, Li Y, Luo M, Yao X, Fan J and Geng J: Circular RNA hsa_circ_0068871 regulates FGFR3 expression and activates STAT3 by targeting miR-181a-5p to promote bladder cancer progression. J Exp Clin Cancer Res 38: 169, 2019.

24. Li X, He M, Guo J and Cao T: Upregulation of circular RNA circ-ERBB2 predicts unfavorable prognosis and facilitates the progression of gastric cancer via miR-503/CACUL1 and miR-637/MMP-19 signaling. Biochem Biophys Res Commun 511: 926-930, 2019.

25. Ambros V: The functions of animal microRNAs. Nature 431: 350-355, 2004

26. Chan JA, Krichevsky AM and Kosik KS: MicroRNA-21 is an antiapoptotic factor in human glioblastoma cells. Cancer Res 65: 6029-6033, 2005.

27. Lei B, Huang Y, Zhou Z, Zhao Y, Thapa AJ, Li W, Cai W and Deng Y: Circular RNA hsa_circ_0076248 promotes oncogenesis of glioma by sponging miR-181a to modulate SIRT1 expression. J Cell Biochem 120: 6698-6708, 2019.

28. Zhou YX, Wang C, Mao LW, Wang YL, Xia LQ, Zhao W, Shen $\mathrm{J}$ and Chen J: Long noncoding RNA HOTAIR mediates the estrogen-induced metastasis of endometrial cancer cells via the miR-646/NPM1 axis. Am J Physiol Cell Physiol 314: C690-C701, 2018.

29. Zhang P, Tang WM, Zhang H, Li YQ, Peng Y, Wang J, Liu GN, Huang XT, Zhao JJ, Li G, et al: MiR-646 inhibited cell proliferation and EMT-induced metastasis by targeting FOXK1 in gastric cancer. Br J Cancer 117: 525-534, 2017.

30. Zhang YS, Chen T, Cai YJ, Dong J, Bai F, Gao X, Tian L, Duan N and Liu D: MicroRNA-647 promotes the therapeutic effectiveness of argon-helium cryoablation and inhibits cell proliferation through targeting TRAF2 via the NF- $\kappa \mathrm{B}$ signaling pathway in non-small cell lung cancer. Onco Targets Ther 11: 6777-6784, 2018.

31. Ye G, Huang K, Yu J, Zhao L, Zhu X, Yang Q, Li W, Jiang Y, Zhuang B, Liu H, et al: MicroRNA-647 targets SRF-MYH9 axis to suppress invasion and metastasis of gastric cancer. Theranostics 7: 3338-3353, 2017. 
32. Ralston E, Beushausen S and Ploug T: Expression of the synaptic vesicle proteins VAMPs/synaptobrevins 1 and 2 in non-neural tissues. J Biol Chem 269: 15403-15406, 1994.

33. Raja SA, Abbas S, Shah STA, Tariq A, Bibi N, Yousuf A, Khawaja A, Nawaz M, Mehmood A, Khan MJ and Hussain A: Increased expression levels of Syntaxin 1A and Synaptobrevin $2 /$ vesicle-associated membrane protein- 2 are associated with the progression of bladder cancer. Genet Mol Biol 42: 40-47, 2019.

34. Li L, Wang X and Liu D: MicroRNA-185 inhibits proliferation, migration and invasion in human osteosarcoma MG63 cells by targeting vesicle-associated membrane protein 2 . Gene 696: 80-87, 2019.

35. Wade M, Li YC and Wahl GM: MDM2, MDMX and p53 in oncogenesis and cancer therapy. Nat Rev Cancer 13: 83-96, 2013 .
36. Vassilev LT, Vu BT, Graves B, Carvajal D, Podlaski F, Filipovic Z, Kong N, Kammlott U, Lukacs C, Klein C, et al: In vivo activation of the p53 pathway by small-molecule antagonists of MDM2. Science 303: 844-848, 2004.

37. Sawada R, Maehara R, Oshikiri T, Nakamura T, Itoh T, Kodama Y, Kakeji Y and Zen Y: MDM2 copy number increase: A poor prognostic, molecular event in esophageal squamous cell carcinoma. Hum Pathol 89: 1-9, 2019.

38. Wang W, Wu J, Fei X, Chen W, Li Y, Shen K and Zhu L: CHD1L promotes cell cycle progression and cell motility by up-regulating MDM2 in breast cancer. Am J Transl Res 11: 1581-1592, 2019.

39. Tang Y, Xuan Y, Qiao G, Ou Z, He Z, Zhu Q, Liao M and Yin G: MDM2 promotes epithelial-mesenchymal transition through activation of Smad2/3 signaling pathway in lung adenocarcinoma. Onco Targets Ther 12: 2247-2258, 2019. 